\title{
Usefulness of ultrasound in the diagnosis of nosocomial maxillary sinusitis in patients with severe Covid-19 pneumonia: a retrospective study
}

\author{
Issac Cheong ${ }^{1,2}$ (1) $\cdot$ Victoria Otero Castro ${ }^{1} \cdot$ Raúl Alejandro Gómez ${ }^{1} \cdot$ Francisco Marcelo Tamagnone $^{2}$
}

Received: 6 December 2021 / Accepted: 10 January 2022 / Published online: 28 January 2022

(c) Società Italiana di Ultrasonologia in Medicina e Biologia (SIUMB) 2022

\begin{abstract}
Purpose Severe Covid-19 pneumonia frequently presents with infective complications as bacterial and fungal infections, nosocomial maxillary sinusitis is one of them. We describe the role of ultrasonography in the diagnosis of nosocomial maxillary sinusitis in patients undergoing mechanical ventilation due to severe Covid-19 pneumonia.

Methods Patients with severe pneumonia due to Covid-19 requiring mechanical ventilation and had maxillary sinus occupation by ultrasonography were retrospectively enrolled at intensive care unit of Sanatorio De los Arcos, Buenos Aires, Argentina, between March 2020 and May 2021.

Results We evaluated a total of 171 patients with severe Covid-19 pneumonia requiring mechanical ventilation during the study period. We included 26 patients that had maxillary sinus occupation by ultrasonography (15.2\%). Out of the 26 patients, in 17 the diagnosis was confirmed by surgical drainage of the maxillary sinus with positive cultures with a positive predictive value of $85 \%$. In $34.6 \%$ of the cases the maxillary sinus disease was unilateral and in $38.4 \%$ bilateral. A sinus computed tomography (CT) was performed in $30.7 \%$ of the patients. Most of the patients underwent to surgical drainage without a CT scan $(66.7 \%)$.
\end{abstract}

Conclusions Ultrasonography is a useful tool for the detection of maxillary sinus infection in patients with severe Covid-19 pneumonia and avoids to perform a CT scan and, therefore, unnecessary transfers.

Keywords POCUS $\cdot$ Ultrasonography $\cdot$ Sonography $\cdot$ Maxillary sinusitis $\cdot$ Covid

\section{Introduction}

Secondary bacterial infections are important risk factors contributing to the severity and mortality of Covid-19 patients. In Wuhan was reported $15 \%$ of secondary infections, with higher incidence among non-survivors [1]. Patients with severe Covid-19 pneumonia requiring mechanical ventilation are more prone to develop nosocomial infections, as maxillary sinusitis [1-4]. The gold standard for maxillary sinus occupation is a sinus computed tomography (CT). The

Issac Cheong

issac_cheong@hotmail.com

1 Intensive Care Unit, Department of Critical Care Medicine, Sanatorio De los Arcos, Juan B. Justo 909, CABA, Buenos Aires, Argentina

2 Argentinian Critical Care Ultrasonography Association (ASARUC), Buenos Aires, Argentina confirmed diagnosis of nosocomial maxillary sinus infection is bacterial growth in cultures or the presence of macroscopic pus during surgical drainage [3].

In order to perform a CT scan, the patient needs to be transfer outside the intensive care unit. Given that in-hospital transfers present frequent adverse effects (up to $71 \%$ ) performing a bedside diagnosis may be safer for patient care and reduce workload [4]. Also when the healthcare system reaches saturations, as in Covid-19 pandemic, strategy must be adjusted to fairly allocate scarce resources, in order to maximize the benefit of the greatest number of patients [5].

Ultrasonography showed to be a useful tool for the diagnosis of maxillary occupation with adequate levels of sensitivity and specificity, in addition to its speed, safety, and reproducibility [6]. We describe our experience regarding the role of ultrasonography in the diagnosis of nosocomial maxillary sinusitis in patients undergoing mechanical ventilation due to Covid-19 pneumonia. 


\section{Materials and methods}

We conducted a retrospective, single-center observational study in the intensive care unit of Sanatorio De Los Arcos (Buenos Aires, Argentina) from March 2020 to May 2021. The study was conducted following the principles of the Helsinki Declaration and within the precautions established by ethical and legal standards, preserving the identity of patients and having obtained written informed consent of the participants for the use of their data.

We included all the adult patients diagnosed with Covid-19 pneumonia admitted to the intensive care unit with mechanical ventilation requirement with suspected infection and an image compatible with maxillary sinus occupation by ultrasonography.

\section{Data collection}

We collected the following variables from the electronic medical record: age, sex, comorbidities, APACHE II score, shock, days of mechanical ventilation, mortality, partial or total sinus occupation, unilateral or bilateral affection, fever, leukocytosis, CT scan sinus infection confirmation, surgical drainage requirement, and antibiotic treatment. We reviewed the stored ultrasonographic studies.

Fig. 1 Image in B mode using a phased array transducer placed in the upper maxillary bone showing total occupation of the maxillary sinus. The anechoic liquid content allows the display of the hyperechoic maxillary sinus walls

\section{Ultrasonographic assessment}

For the ultrasonographic evaluation, we used a Philips Envisor HD or Sparq ultrasound machine (Philips Healthcare, MA, USA) with a phased array S2-4 transducer. We placed the probe in each upper maxillary bone pointing toward right. In normal conditions, the presence of air causes reverberation artifices such as parallel echoes. When the maxillary sinus is fully occupied, we can observe its content (anechoic liquid) surrounded by hyperechoic sinus walls. Partial identification of the posterior wall is compatible with partial sinus occupation [2, 7] (Figs. 1, 2).

\section{Protocol of study}

We performed an ultrasonographic evaluation of both maxillary sinuses in all patients with suspected maxillary sinusitis as a probable nosocomial infection source. In transferable patients, we requested a sinus CT scan. If the patient presented ultrasonographic sinus occupation, a surgical drainage was performed. The definitive diagnosis of maxillary sinusitis was confirmed when there was bacterial growth in cultures in the material obtained during surgical drainage.

\section{Statistical analysis}

The frequencies and distribution of the qualitative variables are expressed in absolute quantity and percentage, while

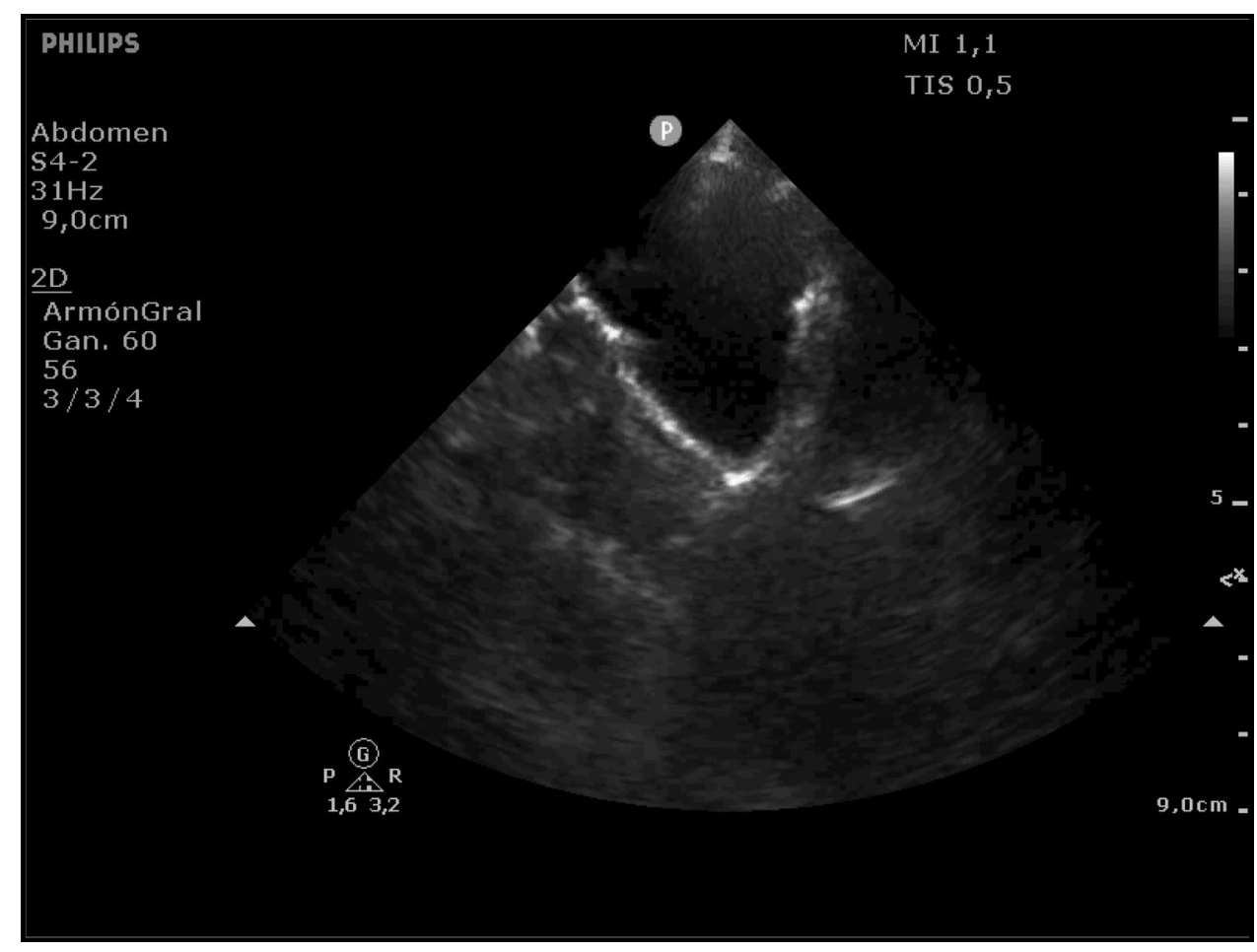


Fig. 2 Image in B mode using a phased array transducer placed in the upper maxillary bone showing partial occupation of the maxillary sinus.

The anechoic liquid content only allows the display of the hyperechoic posterior portion of the maxillary sinus walls

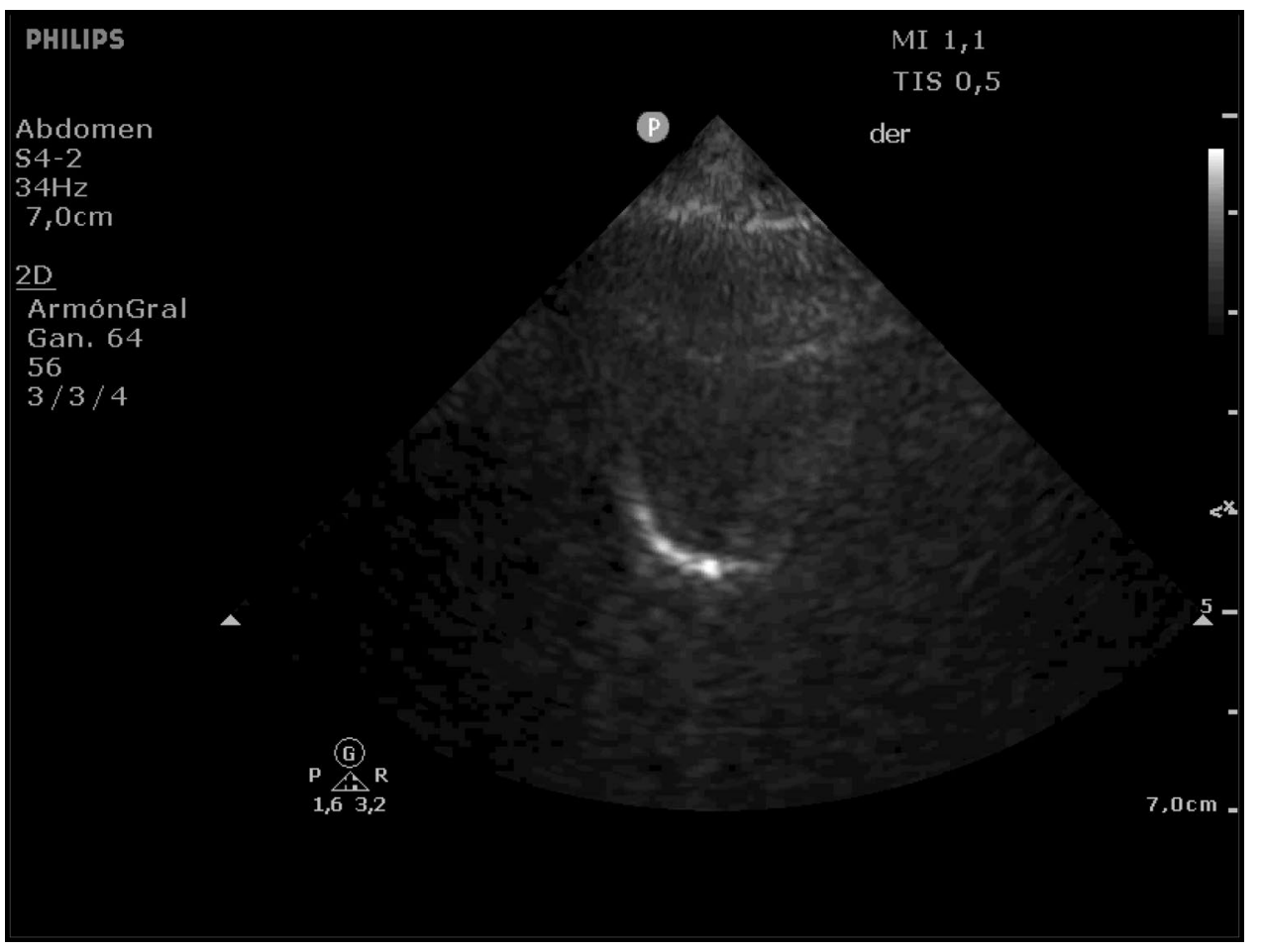

the quantitative variables are presented as mean \pm standard deviations, or median and interquartile range, according to the observed characteristics of the distribution. The normal distribution of quantitative variables was analyzed with the Shapiro-Wilks test. As exploratory analysis we calculated the positive predictive value of ultrasonography for the diagnosis of nosocomial maxillary sinusitis.

\section{Results}

A total of 171 patients with severe Covid-19 pneumonia requiring mechanical ventilation were evaluated during the study period, 26 of them had maxillary sinus occupation diagnosed by ultrasonography (15.2\%). Table 1 describes the demographic characteristic of the patients.
Table 1 Demographic characteristics of patients included in the study

\begin{tabular}{llll}
\hline Variables & All $(N=26)$ & Drained $(N=18)$ & Not drained $(N=8)$ \\
\hline Age (IQR) & $59(49-69)$ & $56(49-72)$ & $67(54-70)$ \\
Male gender & $21(80.7 \%)$ & $14(66.7 \%)$ & $3(33.3 \%)$ \\
Comorbilities & & & \\
Hypertension & $17(65.3 \%)$ & $12(70.6 \%)$ & $5(29.4 \%)$ \\
Dyslipemia & $6(23 \%)$ & $5(71.4 \%)$ & $2(28.6 \%)$ \\
Diabetes & $6(23 \%)$ & $4(66.7 \%)$ & $2(33.3 \%)$ \\
Obesity & $8(30.7 \%)$ & $8(100 \%)$ & 0 \\
Ischemic heart disease & $3(11.5 \%)$ & $1(33.3 \%)$ & $2(66.7 \%)$ \\
Atrial fibrillation & $2(7.6 \%)$ & 0 & $2(100 \%)$ \\
Ex-smoker & $5(19.2 \%)$ & $4(80 \%)$ & $1(20 \%)$ \\
Asthma & $2(7.6 \%)$ & $2(100) \%$ & 0 \\
APACHE II score & $14 \pm 6$ & $13.7 \pm 6.6$ & $14.6 \pm 4.8$ \\
Shock & $6(23 \%)$ & $4(66.7 \%)$ & $2(33.3 \%)$ \\
Days on mechanical ventilation & $24.1 \pm 10.2$ & $26.6 \pm 9.6$ & $21.2 \pm 11.1$ \\
Mortality & $7(26.9 \%)$ & $4(57.1 \%)$ & $3(42.9 \%)$ \\
\hline
\end{tabular}

$I Q R$ interquartil range 
Table 2 Clinical and ultrasonographic characteristics of the patients

\begin{tabular}{lccc}
\hline Variables & All $(N=26)$ & Drained $(N=18)$ & Not drained $(N=8)$ \\
\hline Total and unilateral affection & $9(34.6 \%)$ & $7(26.9 \%)$ & $2(7.7 \%)$ \\
Partial and unilateral affection & $7(26.9 \%)$ & $2(7.7 \%)$ & $5(19.2 \%)$ \\
Bilateral affection & $10(38.4 \%)$ & $9(34.6 \%)$ & $1(3.8 \%)$ \\
Confirmatory sinus CT & $8(30.7 \%)$ & $6(23 \%)$ & $2(7.7 \%)$ \\
Cause of suspicion & & & \\
Fever & $18(69.2 \%)$ & $13(50 \%)$ & $5(19.2 \%)$ \\
Leukocytosis & $11(42.3 \%)$ & $8(30.7 \%)$ & $3(11.5 \%)$ \\
Antibiotic treatment & $23(88.4 \%)$ & $18(69.2 \%)$ & $5(19.2 \%)$ \\
\hline
\end{tabular}

Table 3 Ultrasonographic characteristics of maxillary sinus and diagnosis

\begin{tabular}{lll}
\hline Variables & Confirmed & Discarded \\
\hline Total and unilateral affection & $6(85.7 \%)$ & $1(14.3 \%)$ \\
Partial and unilateral affection & $2(75 \%)$ & $1(25 \%)$ \\
Bilateral affection & $9(90 \%)$ & $1(10 \%)$ \\
\hline
\end{tabular}

In $38.4 \%$ of cases, the maxillary sinus occupation was total and unilateral, $26.9 \%$ partial and unilateral, and $38.4 \%$ bilateral. We performed a sinus CT in $30.7 \%$ of the patients. Most of the patients underwent to surgical drainage without a CT scan (66.7\%).

Of the 26 patients, 18 patients had surgical drainage. Among them, 17 patients presented bacterial growth in the cultures. The surgical drainage was not performed in 8 patients. In the case of 1 patient, the care team decided to treat a different source of infection, 2 patients had coagulopathy and 3 patients were not within the goals of care; therefore these 6 patients were not included in the analysis for diagnostic accuracy. In the other 2 cases with partial occupation, sinusitis was ruled out by a CT scan with evidence of mucosal thickening. Among all surgical drainages, only 1 case of total affection of the maxillary sinus did not develop bacterial growth in culture. The positive predictive value of ultrasonography for the diagnosis of nosocomial maxillary sinusitis was $85 \%$ compared to the gold standard of bacterial growth in cultures in the material obtained during surgical drainage. Patients with partial and unilateral sinus occupation had a positive predictive value of $75 \%$, patients with total and unilateral occupation $85,7 \%$, and those with bilateral occupation $90 \%$. Clinical and ultrasonographic characteristics are described in Tables 2 and 3. The most frequent germs involved were gram-negative bacilli followed by grampositive cocci. Isolated microorganisms are described in more detail in Table 4.
Table 4 Microorganisms isolated from affected maxillary sinuses

\section{Discussion}

The sinus CT scan is the gold standard for the evaluation of maxillary sinus occupation. However, it requires to transfer critically ill patients, which has been shown to be associated with adverse effects and increased workload during the Covid-19 pandemic [4, 8, 9]. Ultrasonography is a useful tool for diagnosis and follow-up in the intensive care unit. It is safe, cost-effective, and can be performed at patient's bedside. Hilbert et al. demonstrated a sensitivity of $100 \%$ and specificity of $96.7 \%$ for the diagnosis of maxillary sinusitis in patients under mechanical ventilation [10]. Our study showed that CT scan was not essential for the confirmation of maxillary sinus occupation and consequently surgical drainage in $66.7 \%$ of cases. In our study, the ultrasonography has a high positive predictive value $(85 \%)$ for the diagnosis of maxillary nosocomial sinusitis, mainly when the occupation of the maxillary sinus is total or bilateral. 
In the context of a pandemic, being able to perform a bedside diagnosis of maxillary sinusitis, without exposing the patient to an in-hospital transfer, may be safer for patient care, lower work burden and cost-effective.

Our research has several limitations, mainly due to the retrospective design. We were not able to control confounders, not all patients with an image of maxillary sinus occupation by ultrasonography had a CT scan or were surgically drained, and it is a single center study. However, it is a novel method to assess maxillary sinusitis and all the ultrasonographic studies were performed by the same operator, limiting an interobserver variability.

\section{Conclusion}

According to our experience, ultrasonography is a useful tool for the diagnosis of nosocomial maxillary sinusitis in patients with severe Covid-19 pneumonia and may avoid unnecessary transfers in critically ill patients.

Author contributions Conceptualization: IC, RAG. Formal analysis: IC, VOC, RAG. Investigation: IC. Supervision: FMT. Writing-original draft: IC. Writing_review editing: IC, VOC, RAG.

\section{Declarations}

Conflict of interest The author declares that they have no conflict of interest.

\section{References}

1. Hughes S, Troise O, Donaldson H, Mughal N, Moore LSP (2020) Bacterial and fungal coinfection among hospitalized patients with COVID-19: a retrospective cohort study in a UK secondary-care setting. Clin Microbiol Infect [Internet]. 26(10):1395-1399
2. Vargas F, Boyer A, Bui HN, Salmi LR, Gruson D, Hilbert G (2007) A postural change test improves the prediction of a radiological maxillary sinusitis by ultrasonography in mechanically ventilated patients. Intensive Care Med [Internet]. 33(8):14741478. https://doi.org/10.1007/s00134-007-0726-8

3. Savolainen S, Pietola M, Kiukaanniemi H, Lappalainen E, Salminen M, Mikkonen P (1997) An ultrasound device in the diagnosis of acute maxillary sinusitis. Acta Otolaryngol [Internet]. 117(sup 529):148-152. https://doi.org/10.3109/00016489709124108

4. Blakeman TC, Branson RD (2013) Inter- and intra-hospital transport of the critically ill. Respir Care 58(6):1008-1023

5. Thomas L, Donat N, Donat A, Pasquier P, Libert N, Schaeffer E, D'Aranda E, Cotte J, Fontaine B, Perrigaul P, Miche F, Muller L, Meaudre E, Bever V (2020) Priorisation of ICU treatments for critically ill patients in a COVID-19 pandemic with scarce resources. Anaesth Crit Care Pain Med [Internet] 39:333-339

6. Puidupin M, Guiavarch M, Paris A, Caroff P, Boutin JP, Le Bivic $T$ et al (1997) B-mode ultrasound in the diagnosis of maxillary sinusitis in intensive care unit. Intensive Care Med [Internet]. 23(11):1174-1175. https://doi.org/10.1007/s001340050476

7. Karantanas AH, Sandris V (1997) Maxillary sinus inflammatory disease: ultrasound compared to computed tomography. Comput Med Imaging Graph [Internet]. 21(4):233-241

8. Harris MA, Adamson HK, Foster B (2021) Computed tomography during the COVID-19 pandemic: a survey of changes to service delivery, working practices and decision-making role of radiographers. J Med Imaging Radiat Sci [Internet]. 52(3):363-373. https://doi.org/10.1016/j.jmir.2021.04.006

9. Akudjedu TN, Mishio NA, Elshami W, Culp MP, Lawal O, Botwe BO et al (2021) The global impact of the COVID-19 pandemic on clinical radiography practice: a systematic literature review and recommendations for future services planning. Radiography 27:1219-1226

10. Hilbert G, Vargas F, Valentino R, Gruson D, Chene G, Bébéar C et al (2001) Comparison of B-mode ultrasound and computed tomography in the diagnosis of maxillary sinusitis in mechanically ventilated patients. Crit Care Med [Internet]. 29(7):1337-1342

Publisher's Note Springer Nature remains neutral with regard to jurisdictional claims in published maps and institutional affiliations. 